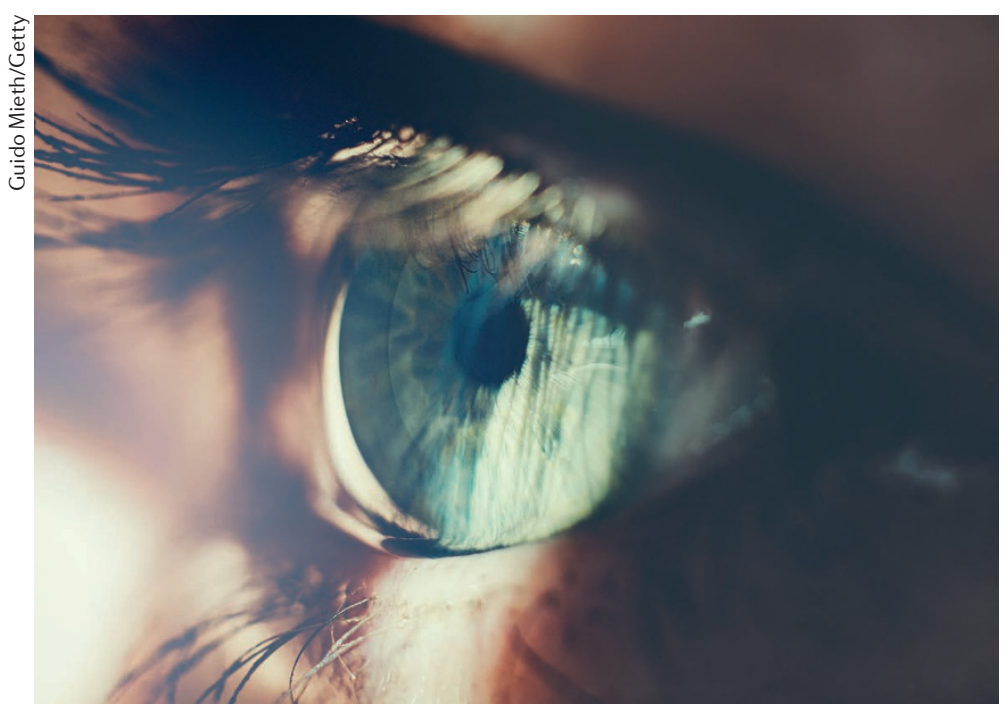

FLEXIBLE ELECTRONICS

\section{Lasting memories are in sight}

The human visual system is capable of detecting, processing and memorizing visual information. Although arrays of sensors have been developed to mimic the human eye, recreating the long-term storage capability of the visual system remains challenging; how can we prevent the image from fading away? Now, writing in Advanced Materials, Di Chen, Guozhen Shen and colleagues report the retention of visual images using integrated UV sensors and resistive switching memory devices.

Resistive memory devices, or memristors, are based on a metalinsulator-metal sandwich structure with a resistance that can be switched between high and low states. The switch from high to low resistance is triggered by an electrical stimulus. Strikingly, the specific resistance state is retained, even after the stimulus has been removed. This memory mechanism is based on the formation and rupture of a conductive filament, governed by the migration of ions from the metal electrode, which is initiated by a positive voltage. Combining this feature of a memristor, to retain resistance information, with a photosensitive material that changes resistance in response to light exposure offers the opportunity to both sense and record applied light.

The researchers fabricated a uniform array of image sensors based on aligned semiconductor micrometre-sized wires using a nearfield direct-printing method. The photoresponse of the sensors to UV light can be adjusted by varying the printing density, thereby enabling a broad and accurate response range. A visual memory array is then realized by integrating the image sensors with memristors, with each pixel consisting of one sensor and one memristor. "When external light is applied on the memory array, the resistance state of the image sensor changes from high to low resistance owing to the light-sensitive oxide semiconductor. The reduction of resistance in the image sensor leads to an increase in partial voltage on the memristor. When the partial voltage reaches the set voltage of the memristor, the resistance switches from OFF to ON," explains Shen. The ON state is maintained through the formation of the conductive filament until a reset voltage is applied. As a result, the incident light on the array is memorized and can be controllably erased, making it possible to store information and then reuse the device.

Using this setup, the researchers demonstrate how the memory device can be used to visualize and memorize complex objects, such as a butterfly, for more than a week. "The light information perceived by the image sensor can be recorded in the non-volatile memory devices, thereby mimicking the detection and memory capacities of the human visual memory," says Chen.

The authors envisage using the artificial visual system in electronic eyes and wearable electronics, and to integrate the system with electronic skin to construct an artificial haptic memory system. "We are looking forward to applying this artificial system in auxiliary equipment for patients with visual impairment, helping them to see the world again, although there are still many challenges to overcome, such as biocompatibility and the connection to the nervous system," comments Shen.

The next step is to expand the sensing range from UV to visible light to better mimic the range of the human eye. In addition, an increase in pixel density is likely to improve the spatial resolution in order to capture and memorize high-resolution images.

Christine-Maria Horejs

ORIGINAL ARTICLE Chen, S. et al. An artificial flexible visual memory system based on an

UV-motivated memristor. Adv. Mater. https://doi. org/10.1002/adma.201705400 (2018) 